\title{
Entanglement transition from variable-strength weak measurements
}

\author{
M. Szyniszewski ${ }^{*}$ A. Romito, and H. Schomerus \\ Department of Physics, Lancaster University, Lancaster LA1 4YB, United Kingdom
}

(Dated: March 14, 2019)

\begin{abstract}
We show that weak measurements can induce a quantum phase transition of interacting manybody systems from an ergodic thermal phase with a large entropy to a nonergodic localized phase with a small entropy, but only if the measurement strength exceeds a critical value. We demonstrate this effect for a one-dimensional quantum circuit evolving under random unitary transformations and generic positive operator-valued measurements of variable strength. As opposed to projective measurements describing a restricted class of open systems, the measuring device is modeled as a continuous Gaussian probe, capturing a large class of environments. By employing data collapse and studying the enhanced fluctuations at the transition, we obtain a consistent phase boundary in the space of the measurement strength and the measurement probability, clearly demonstrating a critical value of the measurement strength below which the system is always ergodic, irrespective of the measurement probability. These findings provide guidance for quantum engineering of manybody systems by controlling their environment.
\end{abstract}

Generic many-body quantum systems obey the eigenstate thermalization hypothesis (ETH) [1-4], according to which they establish the characteristics of thermal equilibrium in a finite time. These systems are characterized by highly entangled eigenstates, i.e., states following an extensive scaling of their entanglement entropy, a behavior known as the volume law. However, systems with local interactions can display a transition to a phase in which the entanglement entropy obeys a subextensive area-law scaling, where ETH is violated. A paradigmatic case is that of many-body localization [5] 8 , in which the entanglement transition 9 12 is driven by the strength of a local disordered potential. The fate of this transition in open quantum systems is the subject of intense current investigations [13 17. A promising path to address the entanglement transition in this setting is to consider a randomly driven system on which the external environment acts as a quantum detector [18 21]. In this scenario, the free unitary evolution of the unobserved system leads to a ballistic temporal growth of entanglement 22 24, until it settles into a highly entangled quasi-stationary state that follows the volume law. This tendency is counteracted by local measurements, which induce a stochastic nonunitary backaction. Very recently it has been shown that when local projective measurements are performed frequently enough one encounters an entanglement transition to a quasi-stationary state characterized by an area law [18, 20.

Projective measurements capture an important but restricted class of environments. A much larger class can be accessed by adopting a more general perspective, according to which quantum measurements are not necessarily characterized by discrete projections in the time evolution, but are generically given by positive operator-valued measurements (POVMs) with a continuous spectrum of outcomes and a corresponding stochastic back-action onto the system [25] 27]. The measurement strength $\lambda$ can then be controlled to continuously interpolate be- tween the unobserved case and projective measurements. Such weak measurements are also routinely employed in experiments for quantum states readout [27] and quantum feedback protocols [28 30, and can be used as a theoretical tool to reproduce Lindblad dynamics of open systems via trajectory averaging [25, 26]. They, therefore, describe a much more versatile framework to model the influence of the environment on an open quantum system.

In this Letter, we study the effect of these weak measurements on the ergodic properties of randomly driven many-body systems, modeled as a quantum circuit with local interactions. By analyzing the entanglement entropy and its variance we show that weak measurements can indeed drive a transition to a nonergodic phase obeying the area law. Similarly to the case of projective measurements [18, the result resembles that of the Zeno effect in which a transition to frozen dynamics is obtained by sufficiently frequent projective measurements, while the corresponding question for less disruptive environments was unclear 21]. Importantly, we identify a minimal measurement strength below which no localization is possible regardless of the measurement probability $p$. These results are obtained by mapping out the phase boundary by two different methods, based on the data collapse and scaling of the entropy for different system sizes, as well as the statistical fluctuations of the entropy, which turn out to be maximal at the phase transition. Beyond providing this specific phenomenology, our results open up a broader avenue to study many-body quantum dynamics in open systems, understand their properties in more detail, and guide the implementation of quantum feedback control for technological applications.

We analyze the entanglement transition in a model consisting of a spatially periodic one-dimensional quantum circuit consisting of a chain of $L$ spins, where $L$ is assumed to be even to allow the partition of the chain 


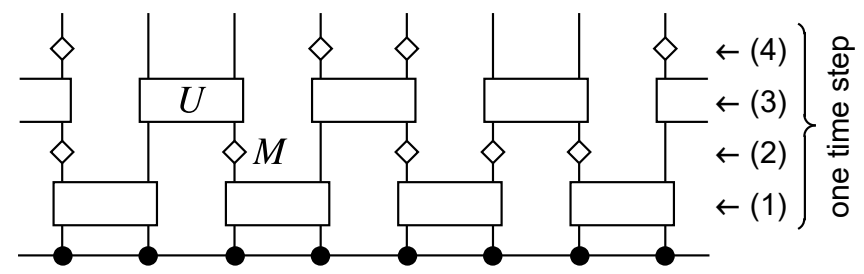

FIG. 1. Diagrammatic representation of the evolution of a weakly measured quantum circuit during one time step. The dots represent the locations of $L$ sites with spin $1 / 2$. The rectangular blocks correspond to unitary operators $U$ on two neighboring spins, while the diamonds correspond to nonunitary evolution induced by the weak measurements $M$ that occur with probability $p$ and have strength $\lambda$. The displayed configuration of diamonds denotes one possible realization of the measurement locations. We consider the dynamics of the system commencing from an unentangled Néel state.

into two subsystems of equal length. The chain evolves in time under a sequence of discrete time steps that are inherently stochastic and consist of a sequence of four operations, as schematically depicted in Fig. 1. First, unitary two-spin operators $U$ are applied between each odd site and the next neighboring site. Each operator is chosen randomly and independently according to the Haar measure over the set of unitary operations for two spins. Second, a measurement $M$ is carried out on each site with probability $p$. In this work, $M$ is a properly normalized Kraus operator (not necessarily a projector) associated with a POVM [25, 31 34], as specified in detail below. Third, analogous to the first step, random unitary two-spin operators $U$ are applied between each even site and the subsequent odd site. Finally, another set of single-site measurements $M$ is carried out, with the same probability $p$ as in the second step. These unitary operators and measurements vary throughout space and from time-step to time-step, rendering the local interactions and measurements disordered and aperiodic. Therefore, both the unitary evolution and the measurement operations are of a stochastic nature.

The weak measurement operations are implemented as follows. Given the state $|\psi\rangle$ of the circuit, a measurement on the $j$-th site of the chain has a stochastic outcome $x \in \mathbb{R}$ drawn from the state-dependent probability distribution [35, 36]

$$
P(x)=G_{\Delta}^{2}(x-\lambda)\left\langle\psi\left|\Pi_{+}^{(j)}\right| \psi\right\rangle+G_{\Delta}^{2}(x+\lambda)\left\langle\psi\left|\Pi_{-}^{(j)}\right| \psi\right\rangle,
$$

where $G_{\Delta}(x)=\exp \left(-x^{2} / 2 \Delta^{2}\right) /\left(\pi^{1 / 4} \Delta^{1 / 2}\right)$ is a Gaussian probability amplitude of width $\Delta$, while $\Pi_{ \pm}^{(j)}=$ $\left(1 \pm \sigma_{z}^{(j)}\right) \bigotimes_{i \neq j} \mathbf{1}^{(i)}$ projects the $j$-th site component of the state onto the spin-up or spin-down subspace. Given the outcome $x$ from the distribution above, the state is then updated as

$$
\left|\psi^{\prime}\right\rangle=\frac{1}{\sqrt{P(x)}}\left[G_{\Delta}(x-\lambda) \Pi_{+}^{(j)}|\psi\rangle+G_{\Delta}(x+\lambda) \Pi_{-}^{(j)}|\psi\rangle\right] .
$$

This update is a stochastic process that depends on the random measurement outcome $x$, which in turn depends on the state $|\psi\rangle$ before the measurement. This choice of the detector model allows us to bridge between the free unitary dynamics of the unobserved system and projective measurements, as controlled by the measurement strength $\lambda / \Delta$ [35, 37]. The case of no measurements corresponds to $\lambda / \Delta=0$, while strong projective measurements are obtained in the limit $\lambda / \Delta \rightarrow \infty$.

To discriminate between ergodic and nonergodic manybody phases we utilize the bipartite entanglement entropy

$$
S=-\operatorname{tr}\left(\rho_{A} \log \rho_{A}\right),
$$

where $\rho_{A}$ is the reduced density matrix of a subchain $A$. This entropy is extensively employed to quantify the entanglement in quantum systems, and its scaling properties with length can be used to determine whether the system is in an ergodic thermal-like phase or a nonergodic localized phase [9-12, 19, 38, 39. Alternative entanglement measures used in the literature include Rényi entropies [18, 20, 21] and mutual information [20, 21, 38]. We focus on the case where the subchain $A$ is of length $L / 2$, which corresponds to cutting the circuit into two halves of equal size. Note that a cut may be commensurate or incommensurate with the unitary operations $U$ in each layer of the circuit, which can result in differences between systems of length $L=4 k$ or $L=4 k+2$. We, therefore, place these cuts randomly.

The stochastic evolution of the entropy over time is illustrated in Fig. 2 The figure displays a colorcoded histogram obtained from 1000 realizations of the stochastic evolution commencing from an unentangled Néel state, and contrasts two cases of different measurement strength while the measurement probability is fixed to $p=0.9$. For the weaker measurement strength $\lambda / \Delta=0.2$ [panel (a)] the entropy increases ballistically over $\sim L / 2$ time steps and then saturates at a large value, which increases linearly with the system size (see the inset). This is the signature of an extensive entropy scaling according to a volume law. The entropy in the quasistationary regime at long times is close to the value in an unobserved system $(\lambda=0$ or $p=0)$, where the average entropy is predicted to be given by [40]

$$
\langle S\rangle=\frac{L}{2} \log 2-\frac{1}{2} .
$$

A qualitatively different behavior is observed for the stronger measurements with $\lambda / \Delta=0.6$ [panel (b)]. There the entropy saturates quickly, and at a value independent of the system size (see the inset). This cor- 

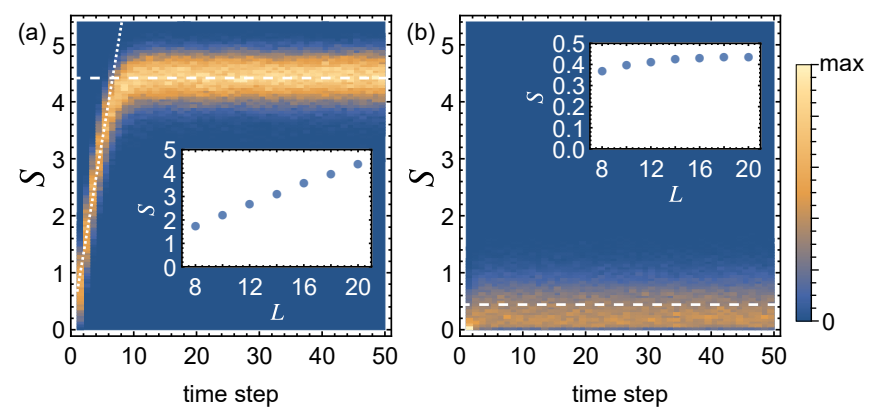

FIG. 2. Color-coded histogram of the bipartite entanglement entropy $S$ for a quantum circuit of length $L=20$ for representative parameters in two regimes, with measurement strength (a) $\lambda / \Delta=0.2$ and (b) $\lambda / \Delta=0.6$ while in both cases the measurement probability is $p=0.9$. The dashed lines indicate the averaged quasistationary values of the entropy after the initial transient growth. The insets show the dependence of these quasistationary values with system size. According to these results, in (a) the system is ergodic and obeys a volume law, while in (b) it is nonergodic and follows an area law. Each panel contains data from 1000 realizations.

responds to an area law, as encountered in a localized phase.

The observation of two phases suggests that a phase boundary exists in the parameter space spanned by $p$ and $\lambda / \Delta$. A clear indication of the support of the phases can be obtained from the variance of the entropy, shown in Fig. 3. We again account for 1000 realizations and collect the data from the quasistationary regime (from $\sim L / 2$ to a cutoff of 100 time steps, much larger than the range of the transient entropy growth for all systems sizes studied here). As before, the initial state is an unentangled Néel state. We observe that there are two regions where the entropy fluctuations are small, separated by a transition region with a pronounced increase of the fluctuations. The contour of maximal variance (thick lines) is stable for different system sizes, while the critical fluctuations along this contour increase with system size, as we further analyze below. The contour indicates that there exists a critical value $p_{\text {crit }}$ for the measurement probability below which there are too few measurements to localize the system, as previously identified in analogous models based on projective measurements (hence $\lambda / \Delta \rightarrow \infty$ ) [18]20]. Departing from the projective measurement scenario, the measurement probability at the transition becomes dependent on the measurement strength, so that measurements have to occur more frequently when the measurement strength decreases. However, even for permanent measurements $(p=1)$, the transition occurs at a finite measurement strength, which we denote as $(\lambda / \Delta)_{\text {crit }}$. Below this measurement strength, the system is always in the ergodic phase. This is our main result. In the remainder of this paper we characterize these transitions in detail, and in particular, confirm that they become

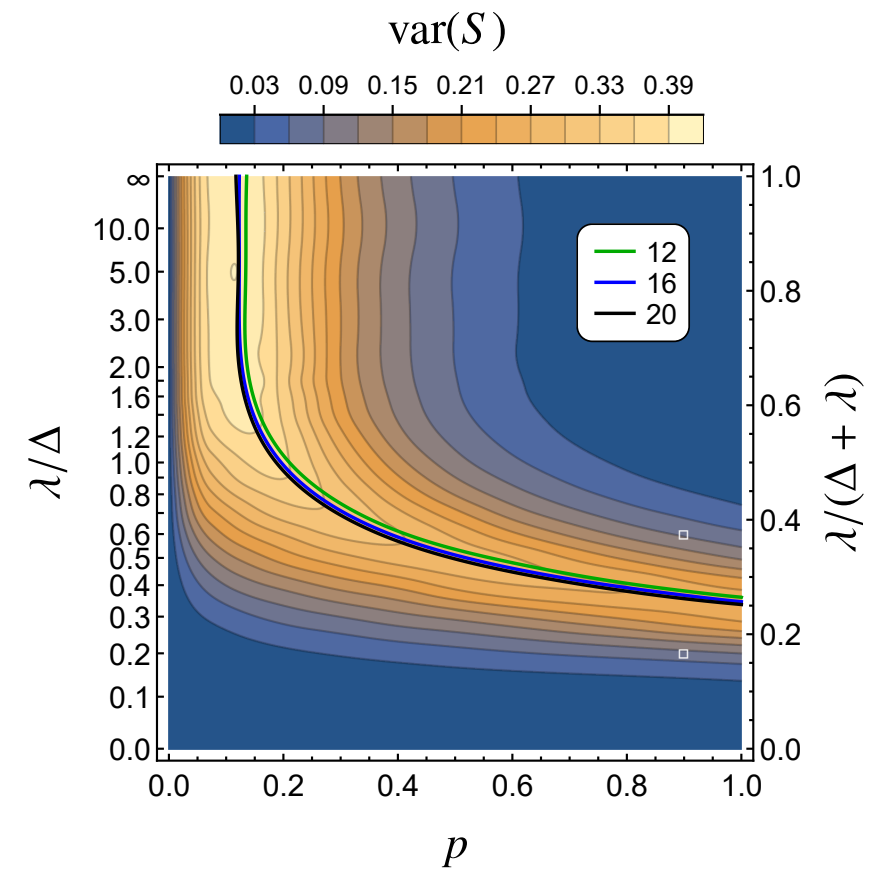

FIG. 3. Color-coded variance $\operatorname{var}(S)$ of the entanglement entropy for varying measurement probability $p$ and the measurement strength $\lambda / \Delta$ in a system of length $L=20$. We observe two regions of small entropy fluctuations, separated by a ridge where the fluctuations are large. The superimposed thick lines show the location of the maximal variance for system sizes $L=12,16,20$, which serves as an indication for the phase boundary (see also the finite-size scaling in Fig. 5 . The two square markers show the two representative points selected for Fig. 2

sharp in the limit of a large system size.

In order to determine the two critical parameters, we consider two distinct transition scenarios: (a) the case where the measurement is always performed $(p=1)$ while the measurement strength $\lambda / \Delta$ is varied, and (b) the case of almost-projective measurements with $\lambda / \Delta=$ 10 and varying probability $p$. Figure 4 displays the average $\langle S\rangle$ of the entanglement entropy for both scenarios. For small $\lambda / \Delta$ or $p$ the entropy clearly follows a volume law, as can be seen by comparison with the theoretical prediction (4) for an unobserved ergodic system. At large values of these parameters, however, the entropy becomes independent of the system size, so that an area law is observed. That we indeed deal with a well-defined transition follows from the finite-size scaling analysis 41, 42] shown in the insets. For this, we assume that in the critical region the correlation length scales as $\xi \sim\left|x-x_{\text {crit }}\right|^{\nu}$ and the entropy scales as $\langle S\rangle \sim\left|x-x_{\text {crit }}\right|^{\gamma}$, where $x=p,(\lambda / \Delta)$ is the varied parameter. The scaling ansatz then takes the form

$$
\langle S\rangle L^{-\gamma / \nu}=F\left(L^{1 / \nu}\left(x-x_{\text {crit }}\right)\right),
$$

where $F$ is an unknown scaling function. The transi- 

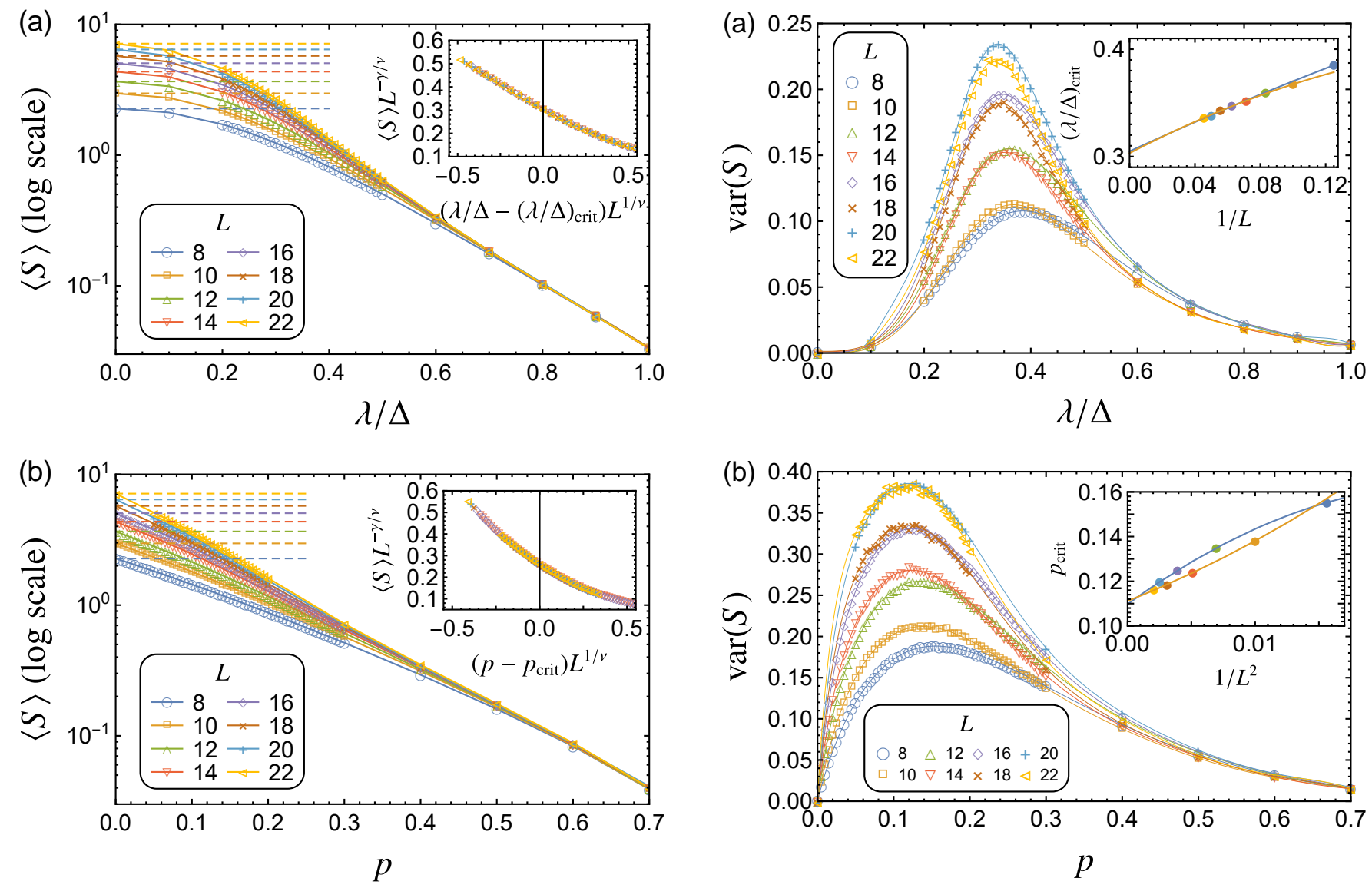

FIG. 4. Comparison of the averaged entropy $\langle S\rangle$ for different system sizes $L$ across the phase transitions with (a) $p=1$ (where measurement are always performed), and (b) $\lambda / \Delta=10$ (where measurements are always strong). The dashed lines show the analytical prediction (4) for an unobserved system. The insets show the finite-size scaling for circuits of length $L \geq 14$. In (a), the data collapse gives the critical measurement strength $(\lambda / \Delta)_{\text {crit }}=0.30(1)$ with critical exponents $\gamma=1.38(7), \nu=1.96(2)$, while in (b) we obtain the critical measurement probability $p_{\text {crit }}=0.110(3)$ with $\gamma=1.94(2), \nu=2.352(5)$. Statistical errors are smaller than the marker size.

tion point $x_{\text {crit }}$ and the critical exponents $\nu$ and $\gamma$ can be obtained by means of a data collapse [43, 44. The data collapse shown in the insets then gives the values $(\lambda / \Delta)_{\text {crit }}=0.30(1)$ and $p_{\text {crit }}=0.110(3)$.

Figure 5 displays analogous results for the entropy fluctuations captured by the variance $\operatorname{var}(S)$. The main panels present the numerical data, along with a fit utilized to enable reliable extraction of the position and height of the maximal variance. We observe that the positions of the maxima display a remarkably small drift with the system size, which reliefs us from the complications encountered in other cases 11, 45. Therefore, we can extract the phase transition directly from finite-size scaling of the locations of the maxima, which is again presented

FIG. 5. Comparison of the variance $\operatorname{var}(S)$ for different system sizes $L$ across the same phase transitions as in Fig. 4 The thin lines are fits that allow reliable extraction of the maxima. The insets show the finite-size scaling of the position of the maxima, where the dark blue line represents the extrapolation for chains of length $L=4 k$, while the light orange line represents the extrapolation for lengths $L=4 k+2$. Statistical errors are smaller than the marker size.

in the insets. We observe two scaling curves, one originating from systems of length $L=4 k$ and another one from systems of length $L=4 k+2$, which, as mentioned before, differ by the commensurability of the bipartition in each layer. Reassuringly, both curves extrapolate to consistent transition points for $L \rightarrow \infty$, giving the values listed in Table [. Combining both transition curves into a simultaneous extrapolation we then obtain the values $(\lambda / \Delta)_{\text {crit }}=0.304(3)$ and $p_{\text {crit }}=0.1103(7)$, in excellent agreement with the values found from the average entropy data collapse.

In conclusion, weak local measurements can drive open many-body systems into a nonergodic low-entropy phase, but only if the measurement strength exceeds a critical value. This means that a continuously observed manybody system can remain ergodic even if the observation strength is finite. We demonstrated our findings for a quantum circuit, evolving under random local unitary op- 


\begin{tabular}{lll}
\hline \hline \multirow{2}{*}{ Method } & \multicolumn{2}{c}{ Critical values } \\
\cline { 2 - 3 } & $(\lambda / \Delta)_{\text {crit }}$ & $p_{\text {crit }}$ \\
& $(p=1)$ & $(\lambda / \Delta=10)$ \\
\hline Data collapse & $0.30(1)$ & $0.110(3)$ \\
Extrap. $(L=4 k)$ & $0.305(5)$ & $0.1097(6)$ \\
Extrap. $(L=4 k+2)$ & $0.303(4)$ & $0.1112(7)$ \\
Simultaneous extrap. & $0.304(3)$ & $0.1103(7)$ \\
\hline \hline
\end{tabular}

TABLE I. Critical values of the measurement strength $\lambda / \Delta$ and the measurement probability $p$ as obtained from the finite-size scaling in Figs. 4 and 5.

erations and local positive operator-value measurements modeled as a Gaussian probe, for which finite-size scaling of the entropy and its fluctuations show that the entanglement phase transition becomes sharp for large systems. Quantum circuits can describe a very large range of dynamics, while weak measurements provide a rather general description of environments, where the variable measurement strength bridges between the fully projective measurements and the fully unitary dynamics of the unobserved system. We, therefore, anticipate that our findings generalize to a large range of settings that deserve further investigation. This includes systems with nonstochastic unitary dynamics as well as different types of interactions, observables, or dimensions, but also practical applications aiming at the control of quantum systems via engineering of their environment.

This research was funded by EPSRC via Grant No. EP/P010180/1. Computer time was provided by Lancaster University's High-End Computing facility.

* mszynisz@gmail.com

[1] J. M. Deutsch, "Quantum statistical mechanics in a closed system," Phys. Rev. A 43, 2046-2049 (1991)

[2] M. Srednicki, "Chaos and quantum thermalization," Phys. Rev. E 50, 888-901 (1994)

[3] L. D'Alessio, Y. Kafri, A. Polkovnikov, and M. Rigol, "From quantum chaos and eigenstate thermalization to statistical mechanics and thermodynamics," Adv. Phys. 65, 239-362 (2016)

[4] F. Borgonovi, F. M. Izrailev, L. F. Santos, and V. G. Zelevinsky, "Quantum chaos and thermalization in isolated systems of interacting particles," Phys. Rep. 626, $1-58(2016)$

[5] D. M. Basko, I. L. Aleiner, and B. L. Altshuler, "Metalinsulator transition in a weakly interacting many-electron system with localized single-particle states," Ann. Phys. 321, 1126-1205 (2006)

[6] I. V. Gornyi, A. D. Mirlin, and D. G. Polyakov, "Interacting electrons in disordered wires: Anderson localization and low-T transport," Phys. Rev. Lett. 95, 206603 (2005)

[7] E. Altman and R. Vosk, "Universal dynamics and renormalization in many-body-localized systems," Annu. Rev.
Condens. Matter Phys. 6, 383-409 (2015)

[8] R. Nandkishore and D. A. Huse, "Many-body localization and thermalization in quantum statistical mechanics," Annu. Rev. Condens. Matter Phys. 6, 15-38 (2015).

[9] M. Žnidarič, T. Prosen, and P. Prelovšek, "Many-body localization in the Heisenberg $X X Z$ magnet in a random field," Phys. Rev. B 77, 064426 (2008).

[10] B. Bauer and C. Nayak, "Area laws in a many-body localized state and its implications for topological order," J. Stat. Mech. Theory Exp. 2013, P09005 (2013)

[11] J. A. Kjäll, J. H. Bardarson, and F. Pollmann, "Manybody localization in a disordered quantum Ising chain," Phys. Rev. Lett. 113, 107204 (2014)

[12] D. J. Luitz, N. Laflorencie, and F. Alet, "Many-body localization edge in the random-field Heisenberg chain," Phys. Rev. B 91, 081103 (2015)

[13] H. P. Lüschen, P. Bordia, S. S. Hodgman, M. Schreiber, S. Sarkar, A. J. Daley, M. H. Fischer, E. Altman, I. Bloch, and U. Schneider, "Signatures of many-body localization in a controlled open quantum system," Phys. Rev. X 7, 011034 (2017)

[14] X. Xu, C. Guo, and D. Poletti, "Interplay of interaction and disorder in the steady state of an open quantum system," Phys. Rev. B 97, 140201 (2018).

[15] M.-T. Rieder, L. M. Sieberer, M. H. Fischer, and I. C. Fulga, "Localization counteracts decoherence in noisy floquet topological chains," Phys. Rev. Lett. 120, 216801 (2018)

[16] J. Marino and R. M. Nandkishore, "Many-body localization proximity effects in platforms of coupled spins and bosons," Phys. Rev. B 97, 054201 (2018).

[17] I. Vakulchyk, I. Yusipov, M. Ivanchenko, S. Flach, and S. Denisov, "Signatures of many-body localization in steady states of open quantum systems," Phys. Rev. B 98, 020202 (2018).

[18] Y. Li, X. Chen, and M. P. A. Fisher, "Quantum Zeno effect and the many-body entanglement transition," Phys. Rev. B 98, 205136 (2018).

[19] A. Chan, R. M. Nandkishore, M. Pretko, and G. Smith, "Weak measurements limit entanglement to area law (with possible log corrections)," (2018), arXiv:1808.05949

[20] B. Skinner, J. Ruhman, and A. Nahum, "Measurementinduced phase transitions in the dynamics of entanglement," (2018), arXiv:1808.05953.

[21] Y. Li, X. Chen, and M. P. A. Fisher, "Measurementdriven entanglement transition in hybrid quantum circuits," (2019), arXiv:1901.08092.

[22] A. Nahum, J. Ruhman, S. Vijay, and J. Haah, "Quantum entanglement growth under random unitary dynamics," Phys. Rev. X 7, 031016 (2017)

[23] C. W. von Keyserlingk, T. Rakovszky, F. Pollmann, and S. L. Sondhi, "Operator hydrodynamics, OTOCs, and entanglement growth in systems without conservation laws," Phys. Rev. X 8, 021013 (2018).

[24] A. Nahum, S. Vijay, and J. Haah, "Operator spreading in random unitary circuits," Phys. Rev. X 8, 021014 (2018).

[25] K. Jacobs, Quantum Measurement Theory and its Applications (Cambridge University Press, 2014).

[26] H. M. Wiseman and G. J. Milburn, Quantum Measurement and Control (Cambridge University Press, 2009).

[27] A. A. Clerk, M. H. Devoret, S. M. Girvin, F. Marquardt, and R. J. Schoelkopf, "Introduction to quantum noise, measurement, and amplification," Rev. Mod. Phys. 82, 
1155-1208 (2010)

[28] R. Vijay, C. Macklin, D. H. Slichter, S. J. Weber, K. W. Murch, R. Naik, A. N. Korotkov, and I. Siddiqi, "Stabilizing Rabi oscillations in a superconducting qubit using quantum feedback," Nature 490, 77-80 (2012), arXiv:1205.5591.

[29] M. S. Blok, C. Bonato, M. L. Markham, D. J. Twitchen, V. V. Dobrovitski, and R. Hanson, "Manipulating a qubit through the backaction of sequential partial measurements and real-time feedback," Nat. Phys. 10, 189193 (2014)

[30] G. de Lange, D. Ristè, M. J. Tiggelman, C. Eichler, L. Tornberg, G. Johansson, A. Wallraff, R. N. Schouten, and L. DiCarlo, "Reversing quantum trajectories with analog feedback," Phys. Rev. Lett. 112, 080501 (2014).

[31] M. A. Nielsen and I. L. Chuang, Quantum Computation and Quantum Information (Cambridge University Press, Cambridge, UK, 2010).

[32] A. Peres, Quantum Theory: Concepts and Methods (Springer, Dordrecht, Netherlands, 2002).

[33] T. A. Brun, "A simple model of quantum trajectories," Am. J. Phys. 70, 719-737 (2002)

[34] A. N. Jordan and A. N. Korotkov, "Qubit feedback and control with kicked quantum nondemolition measurements: A quantum Bayesian analysis," Phys. Rev. B 74, 085307 (2006)

[35] C. M. Caves and G. J. Milburn, "Quantum-mechanical model for continuous position measurements," Phys. Rev. A 36, 5543-5555 (1987).

[36] D. Das and Arvind, "Estimation of quantum states by weak and projective measurements," Phys. Rev. A 89,
$062121(2014)$

[37] Y. Aharonov, D. Z. Albert, and L. Vaidman, "How the result of a measurement of a component of the spin of a spin-1/2 particle can turn out to be 100," Phys. Rev. Lett. 60, 1351-1354 (1988).

[38] J. Eisert, M. Cramer, and M. B. Plenio, "Colloquium: Area laws for the entanglement entropy," Rev. Mod. Phys. 82, 277-306 (2010),

[39] J. H. Bardarson, F. Pollmann, and J. E. Moore, "Unbounded growth of entanglement in models of many-body localization," Phys. Rev. Lett. 109, 017202 (2012).

[40] D. N. Page, "Average entropy of a subsystem," Phys. Rev. Lett. 71, 1291-1294 (1993).

[41] M. E. Fisher, in Critical Phenomena, Proceedings of the 51st Enrico Fermi Summer School, Varenna, Italy, edited by M. S. Green (Academic Press, New York, 1971).

[42] M. E. Fisher and M. N. Barber, "Scaling theory for finitesize effects in the critical region," Phys. Rev. Lett. 28, 1516-1519 (1972).

[43] P. Serra and S. Kais, "Data collapse for the Schrödinger equation," Chem. Phys. Lett. 319, 273 (2000).

[44] K. S. D. Beach, L. Wang, and A. W. Sandvik, "Data collapse in the critical region using finite-size scaling with subleading corrections," (2005), arXiv:condmat/0505194 [cond-mat.stat-mech]

[45] S. Bera, H. Schomerus, F. Heidrich-Meisner, and J. H. Bardarson, "Many-body localization characterized from a one-particle perspective," Phys. Rev. Lett. 115, 046603 (2015) 